\title{
Essais
}

ESSAIS

Revue interdisciplinaire d'Humanités

Hors-série 6 | 2021

Agrobiodiversité et territoires

\section{D'un cheptel conservatoire de races locales à un outil de gestion de la savane : le cas de la chèvre Péï et de la vache Moka à La Réunion}

From a conservatory herd of local breeds to a savannah management tool: the case of the Péi goat and the Moka cow in Réunion

\section{Quentin Rivière}

\author{
(2) OpenEdition \\ Journals \\ Édition électronique \\ URL : https://journals.openedition.org/essais/7734 \\ DOI : 10.4000/essais. 7734 \\ ISSN : 2276-0970 \\ Éditeur \\ École doctorale Montaigne Humanités \\ Édition imprimée \\ Date de publication : 1 mars 2021 \\ Pagination : 125-146 \\ ISBN : 978-2-492780-00-4 \\ ISSN : 2417-4211
}

Référence électronique

Quentin Rivière, " D'un cheptel conservatoire de races locales à un outil de gestion de la savane : le cas de la chèvre Péi et de la vache Moka à La Réunion », Essais [En ligne], Hors-série 6 | 2021, mis en ligne le 16 mars 2021, consulté le 18 janvier 2023. URL : http://journals.openedition.org/essais/7734 ; DOI : https://doi.org/10.4000/essais.7734 


\section{D'un cheptel conservatoire de races locales à un outil de gestion de la savane : Le cas de la chèvre Péï et de la vache Moka à La Réunion}

\section{Quentin Rivière}

L'île de La Réunion se caractérise par une diversité de milieux, d'habitats et d'espèces avec un fort taux d'endémisme. Elle appartient à l'un des 34 points chauds mondiaux de la biodiversité et possède une diversité de paysage exceptionnelle: "Façonnés par le temps, puis par les hommes, ces paysages sont en évolution constante $»^{1}$. L'histoire originaire du peuplement de l'île et la configuration topographique du territoire expliquent en grande partie la concentration sur le littoral de plus de $80 \%$ de la population. L'urbanisation actuelle exerce ainsi de fortes pressions sur les milieux « naturels » littoraux.

Les protections foncières engagées dès les années 1980, par le Conservatoire du littoral et le Département, la création du Parc National (dont le cœur couvre près de $42 \%$ de la surface de l'île) et de la Réserve Naturelle Nationale Marine en 2007, celle de l'étang de Saint-Paul en 2008, témoignent d'une prise en compte par les pouvoirs publics de la nécessité de mettre en place des outils de protection des espaces naturels pour préserver la biodiversité et les paysages réunionnais (Figure 1). En 2010, l'inscription du cœur du Parc National au patrimoine mondial de l'UNESCO assure les objectifs de protection et de préservation de la nature. Cependant force est de constater que la partie la plus protégée de l'île reste le centre. Sur le littoral, plus densément peuplé, on peut aujourd'hui observer une tendance lourde au mitage des espaces naturels et des espaces protégés (Figure 1).

1 Daniel Faure (éd.), Profil environnemental de La Réunion, La Réunion, DEAL, 2013, p. 303. 


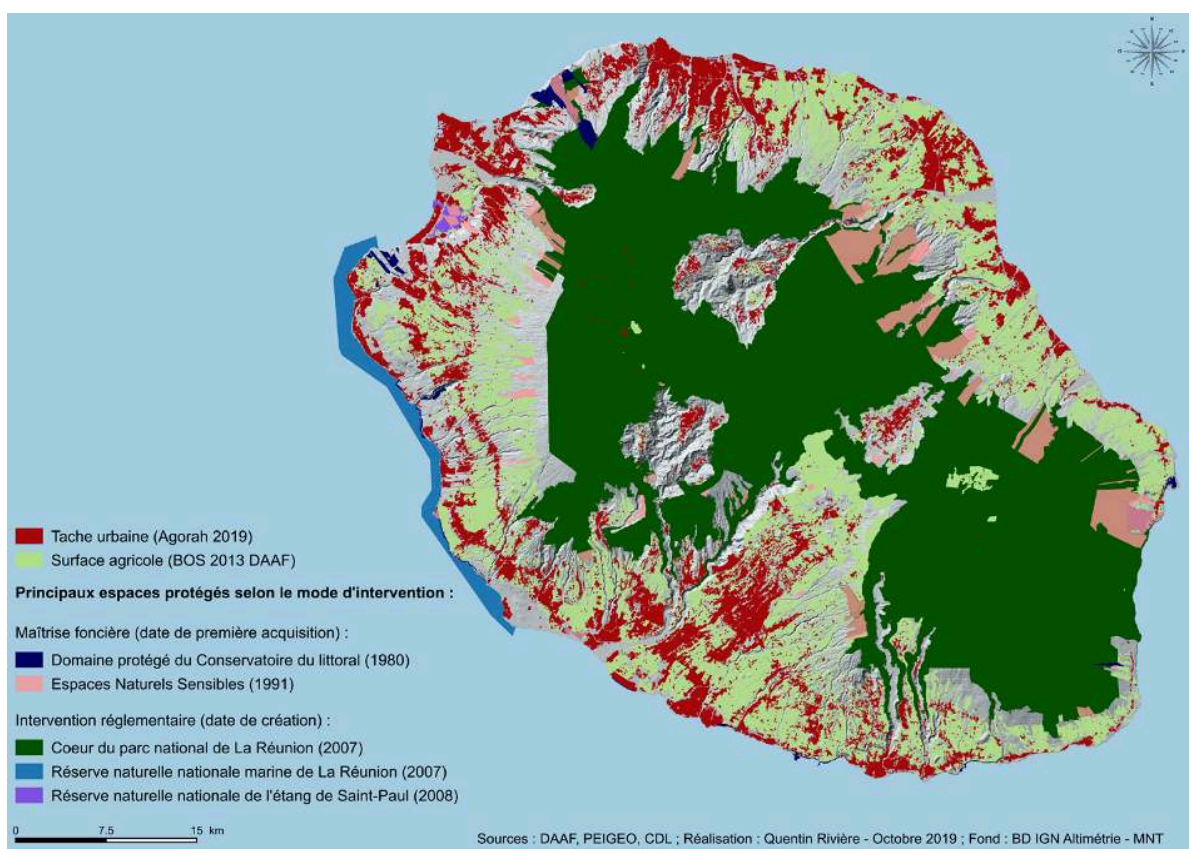

Figure 1 : Une mise en œuvre récente des espaces protégés à La Réunion

(C) Quentin Rivière, 2018

Le littoral ouest de l'île, qui est l'une des régions les plus attractives, connait une pression foncière importante aux dépens des derniers espaces de nature, notamment les espaces de savanes. Pendant longtemps considérés par une partie de la population réunionnaise comme des espaces de friche ou de coupure d'urbanisation, les savanes se révèlent en réalité être des espaces de pratique et de vécu pour d'autres ${ }^{2}$. Au début des années 2000, le Conservatoire du littoral s'intéresse plus particulièrement à la savane du cap la Houssaye. Entre valeurs sociales et enjeux de conservation, nous verrons dans cet article l'intérêt porté à cet espace.

À la même période où certains commencent à s'intéresser au devenir des savanes réunionnaises, d'autres se préoccupent du devenir de la diversité agricole de l'île, notamment celui des races animales locales. La définition d'une race locale (animale) est inscrite dans le Code rural (article D653-9) comme étant " une race majoritairement liée par ses origines, son lieu et son mode d'élevage à un territoire donné (...). Elle présente un intérêt particulier pour la conservation du patrimoine biologique du cheptel ou pour l'aménagement du territoire ». Â La Réunion, il existe deux races animales locales inscrites au registre du Ministère de l'agriculture : la "vache Moka " et la

2 Serge Briffaud (éd.) et al., Les savanes du littoral sous le vent à La Réunion : histoire et dynamiques, perceptions et pratiques, gestion et médiation. Rapport final de recherche pour le Conservatoire du littoral, La Réunion, 2016, p. 209. 
" chèvre Péï ». Ces races sont aujourd'hui reconnues en tant que races à petit effectif et en tant que telles, font l'objet de programmes de sauvegarde. La race à petit effectif est définie dans le Code rural à l'article D653-9 comme une race qui « présente sur le territoire national un effectif de moins de 5000 femelles reproductrices pour les bovins, de 8000 pour les ovins et caprins et de 1000 pour les porcins".

Le sujet développé dans cet article porte sur un projet expérimental qui consiste en la mise en œuvre d'une gestion paysagère par la mobilisation de pratiques telles que le pâturage dirigé et le brûlage dirigé dans la savane du cap la Houssaye, espace aujourd'hui protégé par le Conservatoire du littoral. La définition du brûlage dirigé que nous retiendrons est celle d'Éric Rigolot, qui parle d'une «opération d'aménagement et d'entretien de l'espace comprenant la réduction du combustible sur les ouvrages de prévention des incendies de forêt, ou de gestion des peuplements forestiers, des parcours, des landes et friches. Sur ces zones, le brûlage dirigé consiste à conduire le feu de façon planifiée et ordonnée avec un objectif clairement identifié, sur tout ou partie d'une surface prédéfinie et en toute sécurité pour les espaces limitrophes. Les modes opératoires permettent de maitriser la puissance du feu et de contrôler les impacts sur les différentes composantes du milieu »³. Quant au pâturage dirigé, il s'agit en réalité ici d'une action d'éco-pastoralisme. Ce dernier est défini par Corinne Eychenne qui propose une distinction entre l'éco-pâturage et l'éco-pastoralisme. Ainsi selon l'auteur, "l'éco-pâturage correspond plutôt à un entretien d'espaces verts urbains, généralement en parcs sans surveillance permanente, alors que l'éco-pastoralisme renvoi à la gestion par des troupeaux d'une végétation spontanée, généralement en espace naturel, reposant le plus souvent sur des pratiques associées à la mobilité et au gardiennage $»^{4}$. Le terme pâturage dirigé est utilisé pour faire écho au brûlage dirigé et comprend l'idée que la méthode de pâturage utilisée est contrôlée par un berger qui va conduire le troupeau sur des zones prédéfinies afin d'exercer une pression sur le milieu.

De nombreuses références scientifiques et techniques traitent du pâturage ou del'usage du feu comme modalités de gestion écologique des espaces naturels et des formations herbacées (prairies, zones humides, friches, savanes, etc.). Les discours ne sont cependant pas tranchés. Le feu est parfois perçu comme dévastateur pour les milieux naturels. Selon Michel Vannetier, il « multiplie les risques d'érosion, déclenche une dégradation physique et chimique du sol, provoque la disparition d'une partie des espèces et la multiplication des plus

3 Éric Rigolot, «Le brûlage dirigé en France : outil de gestion et recherches associées ", Prescribed Burning Workshop, Lourizan, Galicia, Espagne, Novembre 1998, p. 13.

4 Corinne Eychenne, "Écopastoralisme et écopâturage : éléments de définition et de discussion ». Rencontres nationales de l'écopâturage organisées par l'Association Entretien, Nature \& Territoire, Saint Herblain, France, Octobre 2014, p. 6. 
adaptées au feu " ${ }^{5}$. Mais lorsqu'il est contrôlé et pour un certain type de milieu, le feu peut être un bon outil de gestion. Johanna Faerber en 1996, explique que le feu peut être un " élément principal de stabilisation et de conservation d'espaces ouverts ${ }^{6}$. S'agissant du pâturage, il semble parfois incompatible avec les ambitions de protection des espaces protégés, notamment dans les aires protégées d'Afrique de l'Ouest comme nous l'explique Jean Boutrais, où la coexistence entre troupeaux domestiques et faune sauvage semble poser des difficultés. Tandis que d'autres ont prouvé que le pâturage peut être un moyen de gestion efficace des milieux et des paysages ouverts ${ }^{8}$. Déjà en 1995, Thierry Lecomte, mettait en avant le pastoralisme dans la gestion des réserves naturelles protégées en France 9 . Les races rustiques et/ou locales sont généralement mises en avant dans ce cas, au regard de leurs capacités d'adaptations au territoire. L'exemple de La Réserve Naturelle Nationale de l'Étang de Cousseau en Gironde et ses vaches de race Marine Landaise, réintroduites en 1990, témoigne bien de ce phénomène.

Dans cet article nous étudierons le cas concret de la mise en place du projet de pâturage dirigé dans la savane du cap la Houssaye. Il s'agira de présenter l'articulation entre un projet de sauvegarde animale de race locale et un projet de gestion paysagère, et de s'interroger sur les conditions de la réussite et de la pérennité des projets. En quoi leurs objectifs respectifs se rejoignent-ils et quels sont les types d'interactions entre les projets et les acteurs? Comment bénéficient-ils du partenariat et comment peuvent-ils fonctionner indépendamment?

\section{De la sauvegarde des races animales locales...}

\section{L'importation d'animaux domestiques agricoles à La Réunion}

Avant l'installation définitive des européens et des malgaches en 1665, l'île servait de point de ravitaillement. Les navigateurs de passage y laissaient des animaux (caprins, bovins, porcins, ovin) comme réserve de nourriture pour

5 Michel Vannetier, "Incendies et forêt : un équilibre précaire ", Espaces Naturels, octobre 2005, $\mathrm{n}^{\circ} 12$, p. 1.

6 Johanna Faerber, « Gestion par le feu et impact sur la diversité : le cas des friches sur anciennes terrasses de culture dans les Pyrénées centrales ", Journal d'agriculture traditionnelle et de botanique appliquée, $38^{\mathrm{e}}$ année, bulletin $\mathrm{n}^{\circ} 1,1996$, p. 273-293.

7 Jean Boutrais, "Chapitre 8. Pastoralisme et aires protégées d'Afrique de l'Ouest en regard de l'Afrique de l'Est ", in Catherine Aubertin et Estienne Rodary (éds), Aires protégées espaces durables?, Marseille, IRD Éditions, 2009, p. 260.

8 "Un paysage est ouvert lorsque la vue peut se développer largement à l'inverse d'un paysage fermé où elle est réduite, interceptée par un écran minéral ou végétal, naturel ou construit : barre d'immeubles, lisière, haie, etc. ", Geoconfluences.ens-lyon.fr.

9 Thierry Lecomte et al., Gestion écologique par le pâturage : l'expérience des réserves naturelles, Réserves Naturelles de France, 1995, p. 82. 
leurs prochaines escales. Des animaux de races et de continents différents ont donc été laissés en liberté sur l'île. Ces animaux se sont développés et se sont adaptés aux conditions physiques et climatiques de l'île ${ }^{10}$. Après les premières installations officielles des habitants, l'introduction d'animaux domestiques agricoles s'est perpétuée. "Un recensement fait en 1709 confirme à la fois l'abondance et la diversité du cheptel réunionnais, même s'il n'est pas aisé de juger de la fiabilité du dénombrement qu'il établit. Alors que la population totale de l'île ne dépasse pas les 900 âmes, on élèverait alors à Bourbon 1991 bœufs, 581 moutons, 1530 cochons, 4529 cabris et 146 chevaux. Le quartier de Saint-Paul nourrit alors l'essentiel des cabris (3 283) et des moutons (456), ainsi qu'une grande partie des bœufs $(904)^{11} »^{12}$. À cette époque d'après les archives de la Compagnie des Indes ${ }^{13}$, le bétail se concentrait dans les Hauts de l'île (les forêts) et sur les espaces littoraux de l'Ouest. La libre circulation et reproduction des animaux ont formé ce que l'on appelle aujourd'hui les races locales. L'élevage se rationnalise au cours du XVIII siècle, avec la construction de parcs, ordonnée par la Compagnie des Indes, pour maîtriser l'élevage. Jusqu'au XIX siècle, les bovins et caprins servent surtout au ravitaillement des navigateurs et de monnaie d'échange pour la population. Au début du $\mathrm{XX}^{\mathrm{e}}$ siècle, avec le développement de la culture de la canne à sucre et les besoins en transport, les bœufs sont utilisés pour tracter les charrettes ${ }^{14}$. L'élevage s'ancre petit à petit dans la culture et le paysage local, et s'incarne par la multiplication de troupeaux à proximité des espaces habités.

Cependant depuis plus de 30 ans on constate un déclin du nombre d'éleveurs et de cheptels bovin de race Moka et caprin de race Péï, et notamment dans les bas de l'Ouest. En cause, nous pouvons évoquer principalement l'impact des modifications des modalités de pratiques d'élevage fondées sur le modèle métropolitain, suite à la départementalisation en 1946. Les filières de production de viande et de lait sont davantage structurées et s'organisent principalement dans les Hauts de l'île, aux pâturages plus verts. La mécanisation de l'agriculture dans les bas et les mi-pentes, incitent certains éleveurs à délaisser leurs pratiques. La représentation du bœuf pour les activités agricoles comme signe d'un bon statut social se perd et l'agriculture mécanisée devient signe de réussite. À la fin du XX siècle, le grand projet ILO de basculement des eaux d'est en ouest, a permis le développement des projets urbains et la conquête agricole sur les derniers espaces « libres » (sans urbanisation) et constructibles

10 Gilles Mandret, L'élevage bovin à la Réunion: Synthèse de quinze ans de recherche, Montpellier, Cirad, 2000, p. 19.

11 Jean Barassin, La vie quotidienne des colons de l'île Bourbon à la fin du règne de Louis XIV, SaintDenis, 1988, p. 211-213.

12 Serge Briffaud (éd.) et al., op. cit., p. 209.

13 Ibid., p. 209.

14 Gilles Mandret, op. cit., p. 19. 
des bas de l'Ouest. Le développement d'infrastructures routières, comme la route des Tamarins construite durant la première décennie des années 2000, a modifié la structure spatiale et la cohérence des pâturages par un morcellement et une réduction des surfaces pâturables.

Enfin, les nouvelles races bovines et caprines importées dans les années 1970 et provenant principalement de France métropolitaine, ont modifié le paysage rural réunionnais et contribué considérablement à la disparition des races bovines et caprines locales. En effet, des races à plus fort rendement (en poids carcasse et en production de lait) ont remplacé progressivement les animaux génétiquement issus des importations antérieures et qui se sont adaptés au territoire. Concernant les cheptels caprins, des races comme la chèvre Boer provenant d'Afrique du Sud, importée en 1976 à La Réunion, la chèvre Alpine originaire du massif alpin ou encore la chèvre Saanen de la vallée de la Saane en Suisse importée à partir des années 1980, ont été croisées et/ou ont remplacé la chèvre locale. En effet, " l'intégration de la chèvre Boer aux qualités bouchères supérieures à la fin du $\mathrm{XX}^{\mathrm{e}}$ siècle a contribué à l'absorption d'une grosse partie du cheptel Péï ${ }^{15}$. Concernant les bovins, les deux principales races importées sont la Limousine et la Blonde d'Aquitaine. "Au début des années 1990, on dénombrait 5000 vaches allaitantes. En 2014, le cheptel est constitué de 9000 vaches allaitantes, dont plus de la moitié sont issues de croisement. Les races Limousine et Blonde d'Aquitaine dominent, qu'elles soient en race pure ou croisée. Parallèlement à la hausse des effectifs, l'amélioration continue de la productivité des troupeaux a impliqué des choix stratégiques. Ainsi, les races qui composent le cheptel actuel se sont substituées progressivement aux bœufs "Moka ", rustiques, issus de croisements entre des zébus malgaches et d'autres races bovines " ${ }^{16}$. À La Réunion, on parlera de «bœufs " pour désigner les bovins et de " cabris " pour désigner les caprins, ce sont là des appellations génériques locales.

\section{La reconnaissance des races animales locales}

Face au constat du déclin de ces races, des programmes de sauvegarde ont été initiés localement. Au début des années 2000 débute un programme de sauvegarde de la chèvre Péï, reposant sur la mise en place d'un standard de la race et d'un recensement de la population en ayant pour objectif la reconnaissance officielle de la race au niveau national. Cette opération est financée par l'Office de Développement de l'Économie Agricole d'outre-mer (ODEADOM). Mais il a fallu attendre 2010 et la création de l'Association

15 Site officiel de CAPGENES : https://www.capgenes.com/les-races-caprines/la-chevre-pei/.

16 Philippe Simon (éd.), Gil Chaulet, Julie Leung, « La production de viande bovine à La Réunion », Agreste DAAF La Réunion, 96, août 2015, p. 7. 
Réunionnaise des Sélectionneurs en Élevage Caprin (ARSEC), pour poursuivre l'opération, cette dernière devenant responsable du suivi des races caprines à La Réunion.

C'est donc au cours de la deuxième décennie des années 2000 que les races locales réunionnaises caprines et bovines ont été reconnues par le ministère de l'agriculture. Le travail de l'ARSEC à partir de 2010 a permis à la chèvre Péï (Figure 2) d'être admise officiellement par le Ministère de l'Agriculture en 2011 par l'arrêté du 22 décembre 2011 modifiant l'arrêté du 26 juillet 2007 " fixant la liste des races des espèces bovines, ovines, caprines et porcines reconnues et précisant les ressources zoogénétiques présentant un intérêt pour la conservation du patrimoine génétique du cheptel et l'aménagement du territoire ». En 2015, avec le financement par le "Fonds Européen Agricole pour le Développement Rural (FEADER) " dans le cadre du programme "Liaison Entre Action de Développement et de l'Économie Rurale (LEADER) » et la participation du Conseil Départemental, l'APPER a monté un dossier de demande de reconnaissance officielle de la race Moka auprès de la Commission Nationale d'Amélioration Génétique (CNAG) et, en 2016, la vache Moka (Figure 3) a été officiellement reconnue par l'arrêté du 13 avril 2016 modifiant l'arrêté du 25 avril 2015 au même titre que la chèvre Péï.

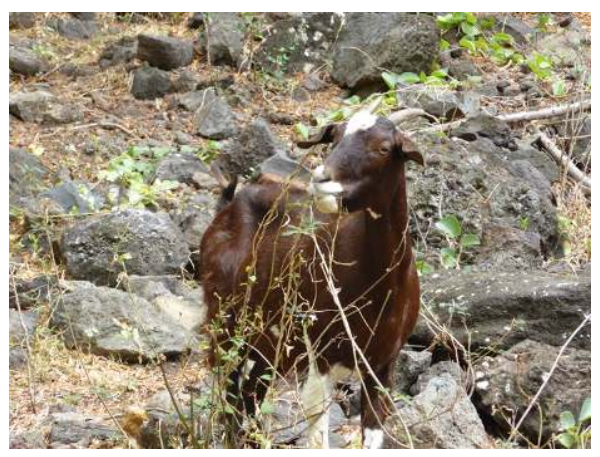

Figure 2 : Chèvre Péi, Saint-Paul (C) Quentin Rivière, 2018

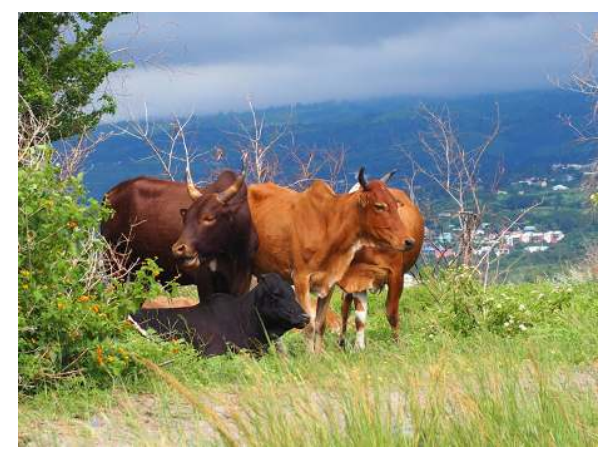

Figure 3 : Vaches Moka, Saint-Paul (C) Lucie Fourthon, 2019

Aujourd'hui, les effectifs dans les registres officiels font état de 600 têtes reproductives de vaches Moka et de 1000 têtes de chèvres Péi à La Réunion ${ }^{17}$.

L'Association pour la promotion du patrimoine et de l'écologie à La Réunion : la volonté d'agir

Créée en 2013, l'APPER est une association loi 1901 à but non lucratif. L'association est investie dans la préservation et le développement local des pratiques et des activités anciennes et traditionnelles. L'association s'intéresse

17 CIRAD - Chambre d'agriculture de La Réunion. 
particulièrement au savoir-faire "lontan ${ }^{18}$ et met en avant des alternatives architecturales, agricoles et écologiques basées sur ces savoir-faire locaux. "L'Association pour la Protection du Patrimoine et de l'Écologie à la Réunion accorde beaucoup de valeur à la notion de "patrimoine ". Celui-ci se décline sous une pluralité de formes. Il est aussi bien culturel, historique, naturel, artisanal, qu'architectural. Une grande partie de nos activités porte sur l'environnement (la nature, l'élevage, l'agriculture biologique) $»^{19}$.

Partant du principe que la faune tient une place privilégiée dans la société réunionnaise, l'association travaille à valoriser les races et les systèmes locaux de production. Depuis plus de 5 ans, l'APPER porte les projets de sauvegarde et de promotion des races animales locales, incarnant à la fois un patrimoine historique, culturel, agricole et génétique à La Réunion. En 2017, grâce aux démarches de l'APPER, la vache Moka obtient le second prix National de la Fondation du Patrimoine pour l'agro-biodiversité animale. Ce prix permet à l'APPER d'obtenir un budget de 6000 euros pour soutenir le plan de sauvegarde de la race. En 2019, l'APPER est mandatée par Capgènes (organisme et entreprise de sélection caprine multiraciale) pour la mise à jour de l'inventaire zootechnique de la race " chèvre Péï ", la récolte de données techniques sur la race et la tenue d'un livre généalogique. L'APPER a donc constitué un troupeau de chèvre Péi qui permettra de donner une meilleure visibilité de la race et de sensibiliser le public à sa sauvegarde.

La reconnaissance officielle des races animales locales est un premier pas dans le projet de sauvegarde porté par l'APPER. Mais l'association œuvre également aujourd'hui à l'accompagnement des derniers éleveurs de vaches Moka et de chèvres Péï qui souhaiteraient développer leur activité et promouvoir les races locales. Elle met en place des réunions d'éleveurs, des groupes de discussion autour des actions prioritaires à mettre en œuvre pour préserver les races locales. Recenser et décrire les races locales, consolider les troupeaux existants et valoriser le travail des éleveurs, tels sont les objectifs de l'association aujourd'hui.

Dans ce sens, depuis 2017, dans le cadre du programme FEADER 2014-2020, l'APPER est en charge de mener une mission d'animation des "Mesures Agro-environnementales et climatiques (MAEC) » à La Réunion. Cette mission consiste à communiquer, sensibiliser et informer les éleveurs sur les MAEC, à accompagner ces derniers dans leur démarche d'engagement et à animer un groupe de travail pour l'adaptation de la MAEC Protection des Races Menacées à La Réunion, avec la conception d'un bulletin d'information et de flyers sur l'intérêt de cette action.

Aujourd'hui l'APPER est un acteur essentiel dans la sauvegarde, la promotion et la valorisation des races animales locales à La Réunion et le développement des activités d'élevage autour de ces races animales locale (Figure 4).

18 Expression locale pour parler des savoir-faire d'antan

19 Site web de l'APPER : http://apper.fr/. 


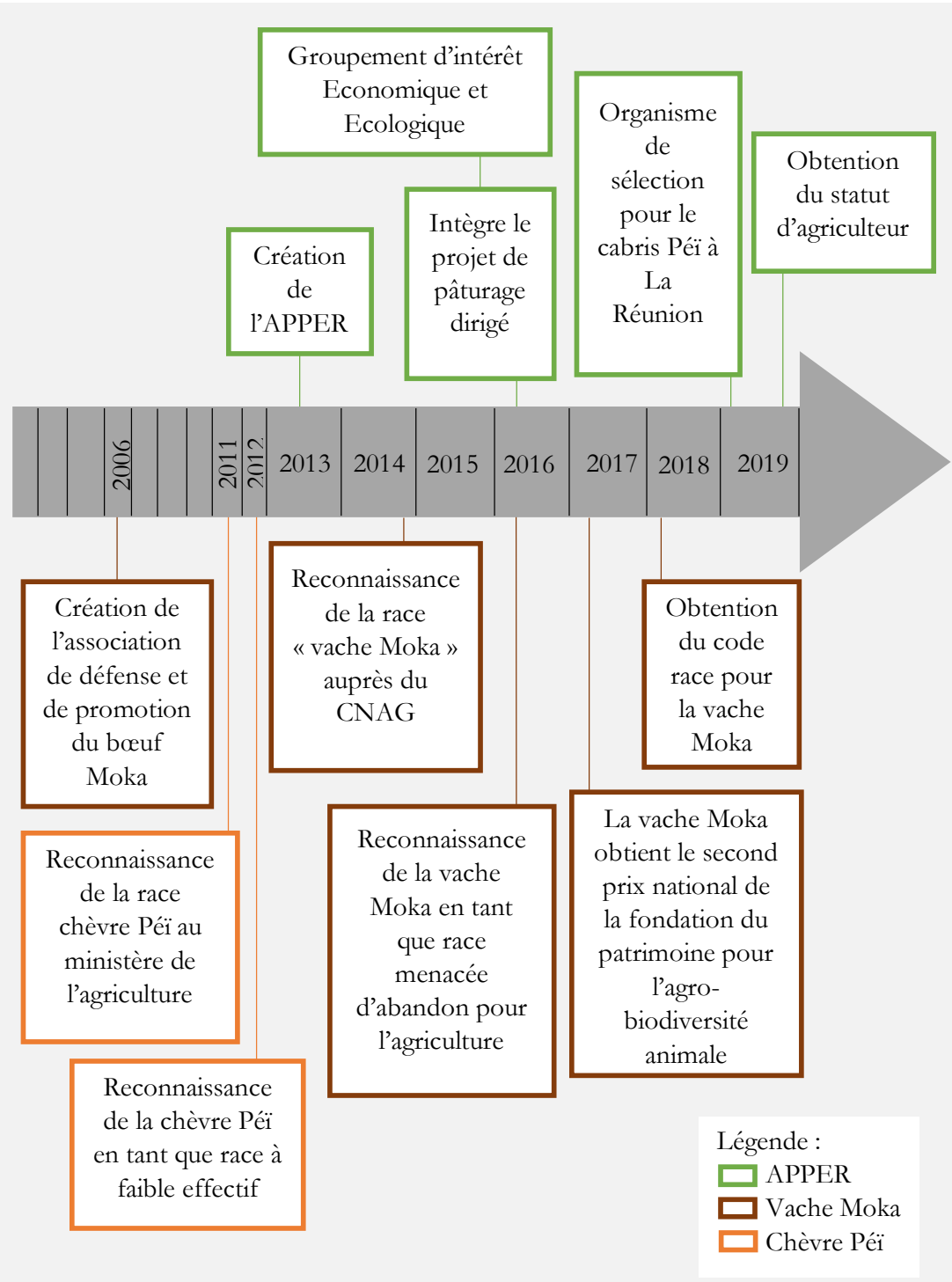

Figure 4 : Chronologie des événements ayant conduit à la protection juridique de la vache Moka et de la chèvre Péi

(C) Quentin Rivière, 2020 


\section{... à l'expérimentation pyro-pastorale dans la savane du cap la Houssaye}

Les savanes réunionnaises : des territoires d'élevage en sursis

Lorsque nous évoquions le sujet des pratiques d'élevage sur le littoral ouest avec les éleveurs, ils rapportaient cette pratique à l'espace de savane. Ils insistaient sur l'étendue des pâturages et le nombre d'éleveurs et de troupeaux qui y pâturaient comme en témoigne ci-dessous l'extrait d'un entretien réalisé en 2017 auprès d'un éleveur de bœuf Moka sur le littoral Ouest.

Q. Rivière : "Ce que j'aimerais savoir c'est comment c'était avant? Comment c'est maintenant?"

Éleveur de Moka : "Avant, avant le pâturage était grand, il n'y avait pas toutes ces maisons-là." "

Q. Rivière : "C'était comment ? C'était comme ici là ? (En montrant la savane)"

Éleveur de Moka : "Partout ici, il n'y avait pas de maisons, tout ça c'était le pâturage pour les boufs. Il n'y avait rien jusqu'au Fond de Cap là-bas. Où il y a le pont. Ça sappelle Fond de Cap, la petite ravine. Par ici, il y avait des animaux, l'établissement avait 30 boufs. Aujourd'hui on les lâche ici, demain là-bas, après demain par là-bas. Chacun à son numéro."

Q. Rivière : "Donc il y avait plusieurs éleveurs?"

Éleveur de Moka : "Ah il y en avait pleins! Là comme je surveille mes boufs, l'autre éleveur surveille ces boufs par là-bas, pour pas quils se bagarrent entre eux. "

Les savanes semblent être considérées par les éleveurs comme le berceau des races bovines et caprines locales. Cela peut être corrélé aux données récoltées lors des recherches réalisées, dans les sources écrites et iconographiques qui démontrent l'existence de troupeaux dans les bas de l'Ouest ${ }^{20}$.

Le littoral ouest de l'île, a été le lieu d'arrivée et d'installation des premiers habitants. Les animaux domestiques agricoles étaient lâchés au large de la baie de Saint-Paul, pour rejoindre la côte, puis conduits vers les grandes propriétés sur les basses pentes pour pâturer ${ }^{21}$. Le pâturage semblait alors être pratiqué de manière extensive avec des parcours dans les espaces, selon les éleveurs interrogés. La composition végétale et les formes paysagères sur le littoral ouest de l'île avant l'arrivée des premiers habitants n'étaient probablement pas les mêmes qu'aujourd'hui, mais nous pouvons supposer que les actions de défrichement, le pâturage et les conditions climatiques ont été favorables à la formation de que l'on appelle aujourd'hui les savanes.

20 Serge Briffaud (éd.) et al., op. cit., p. 209.

21 Saint-Denis : Ars Terres Créoles, in Serge Briffaud (éd.) et al., op. cit., 1989, p. 209. 


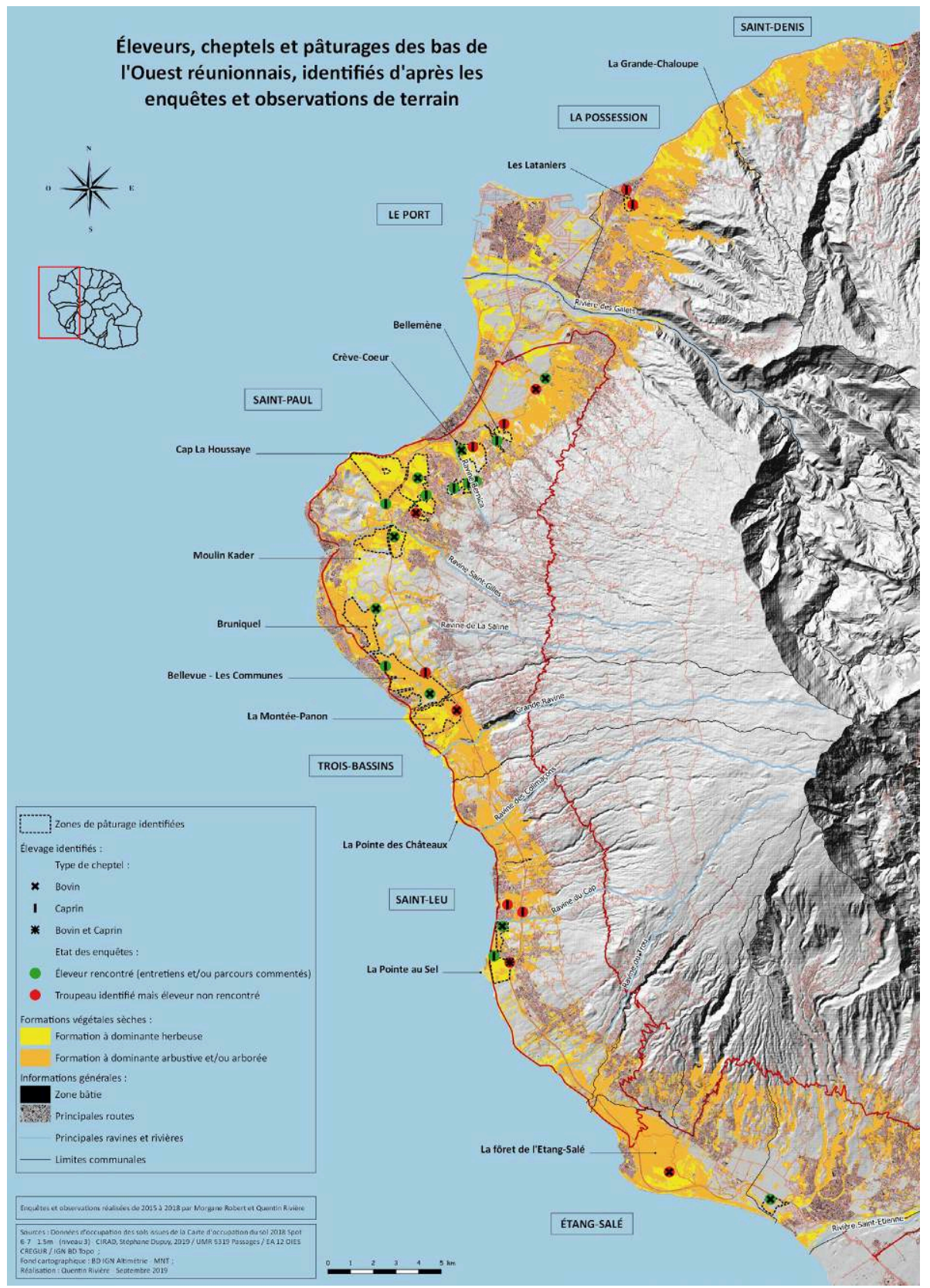

Figure 5 : Éleveurs, cheptels et pâturages des bas de l'ouest réunionnais, identifiés d'après les enquêtes et observations de terrain

(C) Quentin Rivière, 2019

De nos jours, la savane constitue l'un des derniers espaces de pâturage extensif dans les bas de l'ouest (Figure 4). En plus de leurs fonctions pastorales, les savanes acquièrent aujourd'hui des valeurs supplémentaires. Elles « consti- 
tuent le premier niveau de l'une des constructions paysagères étagées les plus spectaculaires de la planète et d'un versant qui forme une unité écologique, aux composantes écosystémiques et paysagères imbriquées et interdépendantes. C'est à ce titre, pour une large part, que les savanes participent à une géodiversité et à une biodiversité qui ne peut être évaluée seulement milieu par milieu, paysage par paysage, mais qui résulte de la cohabitation et de la coévolution, en un espace restreint, de milieux et de paysages contrastés. ${ }^{22}$. Au-delà des considérations paysagères et écologiques associées aux savanes, ces mêmes études ont permis de mettre en avant qu' « elles sont depuis longtemps, pour les habitants des quartiers riverains, le prolongement de l'espace domestique, un lieu de glanage et de cueillette, où l'on a ses petits coins. Elles sont par excellence un espace de liberté, marqué par des pratiques que l'on pourrait nommer "d'exo-sociabilité »-autrement dit des pratiques plus ou moins maronnes, solidaires de l'existence de cet ailleurs proche. Les savanes sont par conséquent aussi le conservatoire d'un mode de vie, le reflet de ce qu'ont de spécifique, dans cette île, les rapports sociaux et le rapport des hommes à l'espace. ${ }^{23}$.

Cependant ces espaces connaissent depuis plus d'une quinzaine d'années des mutations paysagères (Figures 6 et 7) où les paysages, jusqu'alors " ouverts ", c'est-à-dire de formations végétales à dominance herbacée de type graminéen, se ferment peu à peu avec l'envahissement par des espèces ligneuses exotiques ${ }^{24}$. Ces arbustes, épineux pour la plupart, constituent des fourrés continus sur l'espace et contribuent à la fermeture du paysage et ainsi à la formation d'une entrave à l'accessibilité du site. Ces espaces sont également soumis à une pression urbaine et agricole importante dans l'ouest de l'île depuis plus d'une vingtaine d'années, participant à leur disparition progressive.
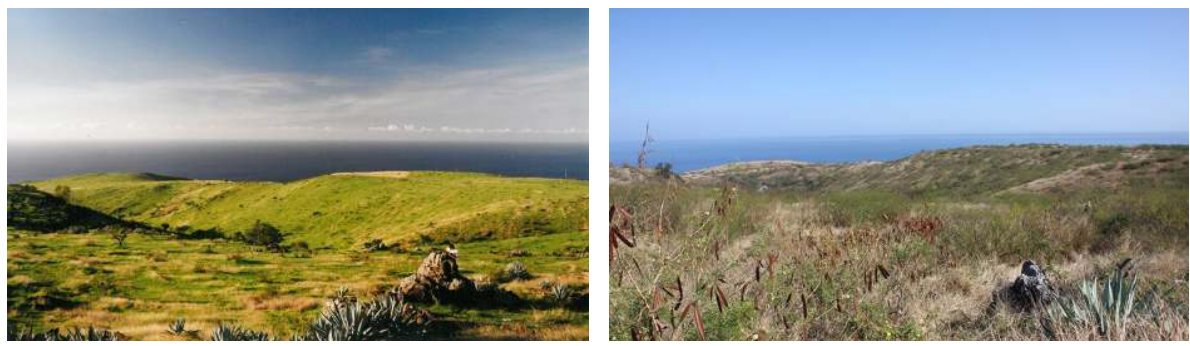

Figures 6 et 7 : Observation du processus d'envahissement par des ligneux exotiques dans la savane du cap la Houssaye entre 2001 et 2016 (Ravine Boucan Canot)

(C) Alexandre Moisset, 2001 et Bernard Davasse, 2016

22 Ibid., p. 209.

23 Ibid., p. 209.

24 Notamment le Leucaena leucocephala (appelée localement Cassi ou Mosa) ou le Vachellia farnesiana (appelée localement Zacassi ou Zépinar). 


\section{Objectifs, acteurs et méthodes expérimentales}

Depuis 2015, le Conservatoire s'est rapproché du Centre National de la Recherche Scientifique (CNRS) ${ }^{25}$ et de l'Université de La Réunion ${ }^{26}$ qui mènent des études sur les savanes réunionnaises dont les objectifs sont de réaliser un état des lieux des savanes afin de consolider les connaissances sur l'histoire, les dynamiques et les usages de ces milieux, de mener une réflexion sur les modalités de conservation et de gestion de ces espaces et enfin de réaliser des expérimentations de gestion sur la savane du Cap la Houssaye ${ }^{27}$. Ces travaux de recherche-action sur les savanes réunionnaises sont menés avec le concours d'associations (APPER, Amis de l'Océan Indien) et d'acteurs institutionnels locaux (Protection Civile, Commune de Saint-Paul, Services départemental d'incendie et de secours, Office national des forêts).

Le Conservatoire du littoral créé en 1975, est un organisme public, chargé de mener une politique de protection foncière de l'espace littoral français aux dépens de l'urbanisation. Il définit des actions de gestion et de conservation viables et durables des sites dans un objectif d'ouverture au public. La gestion des sites est par la suite confiée à un tiers (des collectivités territoriales ou des associations) qui est chargé de l'animation, du suivi des projets de conservation, de la sensibilisation du public, de l'entretien et de la valorisation des sites pour leurs richesses écologiques, patrimoniales et paysagères.

Depuis 2003, le CDL mène une action de maitrise foncière sur le site de la savane du Cap la Houssaye (Figure 8). Il est aujourd'hui propriétaire de 221 ha sur environ 400 ha du site, les autres parcelles étant des propriétés communales ou privées. Le site de la savane du Cap la Houssaye représente des enjeux forts, tant en termes écologiques et paysagers qu'en termes sociaux, identitaires et patrimoniaux ${ }^{28}$. Les études ont permis "d'apporter un éclairage indispensable à la connaissance de ce paysage des savanes en mettant en lumière sa relation à des processus environnementaux (socio-écologiques) inscrits dans la durée et à des pratiques sociales qui contribuent à sa construction/transformation, mais aussi en éclairant sa face immatérielle, sa dimension symbolique et identitaire, les valeurs - elles-mêmes inscrites dans la durée que le paysage revêt pour les populations ».

25 Unité Mixte de Recherche 5319 Passages - GEODE : Géographie de l'Environnement.

26 OIES CREGUR : Océan Indien : espaces et sociétés Centre de Recherches et d'Études en Géographie de l'Université de La Réunion / UMR PVBMT : Unité Mixte de Recherche peuplement végétaux et bioagresseurs en milieux tropical -UMR SELMET : Unité Mixte de Recherche Systèmes d'élevage méditerranéens et tropicaux.

27 Serge Briffaud (éd.) et al., Perceptions de la savane par la population réunionnaise et mise en cuvre d'une gestion des paysages au Cap la Houssaye, Rapport final de recherche pour le Conservatoire du littoral, 2018, p. 191.

28 Serge Briffaud (éd.) et al., op. cit., p. 191. 


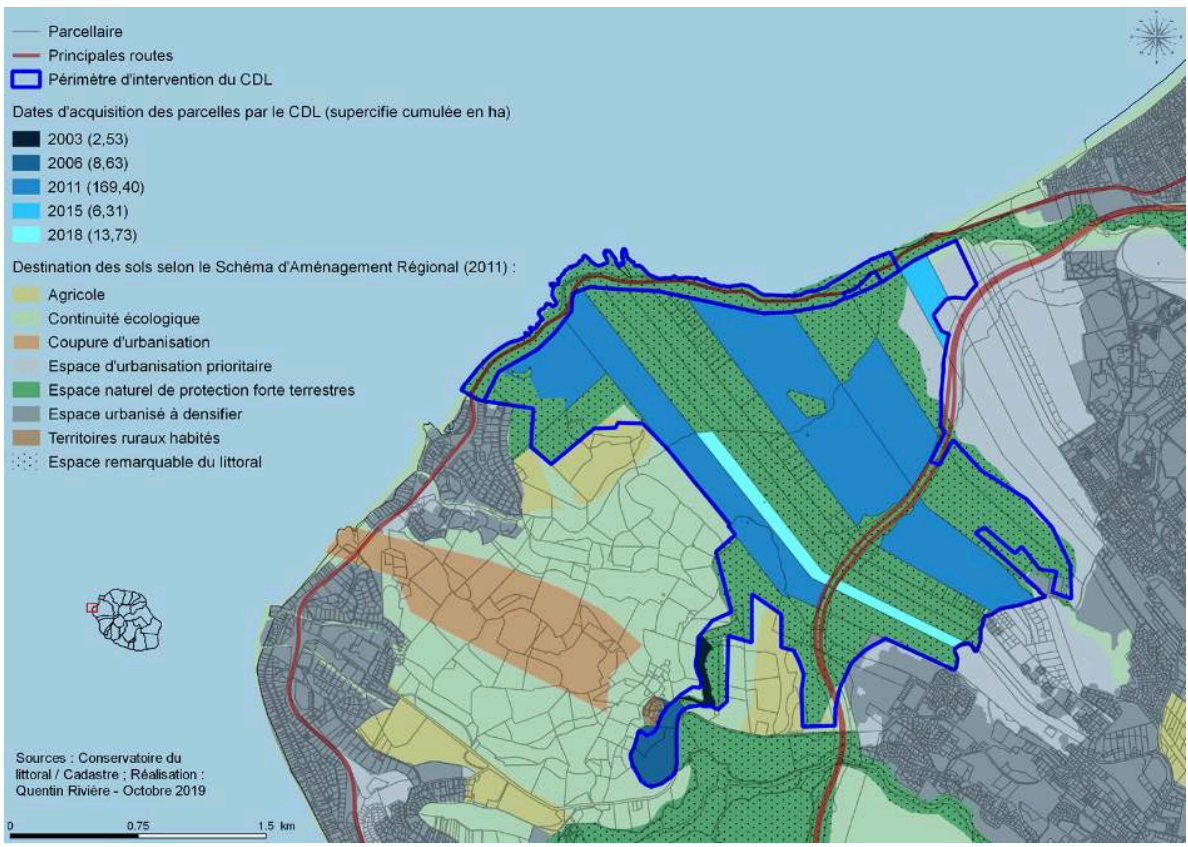

Figure 8 : L'intervention foncière du Conservatoire du littoral sur la savane du cap la Houssaye (C) Quentin Rivière, 2019

Ces enjeux amènent le Conservatoire du littoral à se questionner sur le devenir de cet espace, un devenir qui n'est que dicté par le choix d'agir ou non. D'un paysage subi on passe alors à un paysage voulu, qui nécessite d'orienter les dynamiques en cours. L'objectif étant ici de limiter les effets problématiques de l'expansion des arbustes. Dans la savane du Cap la Houssaye, cette modification de nature du couvert végétal provoque selon le Conservatoire du littoral une altération de la valeur paysagère du site dont la typicité est remise en cause ; une altération de la valeur biologique de cet habitat en tant que tel mais également en tant que contributeur à la connexion écologique des habitats naturels de la commune de Saint-Paul : " la restauration de la savane (espèces indigènes) permettrait d'établir une connexion de meilleure qualité avec les bas de ravines contenant les reliques de végétation indigène ${ }^{29}$; une altération de l'usage de la savane et de ses sentiers qui, du fait de la prolifération des espèces arbustives, se ferme par endroit avec un véritable barbelé végétal.

Le choix du Conservatoire du littoral pour le site du Cap la Houssaye, dont le processus d'envahissement est en cours, s'oriente donc vers le maintien des espaces herbacés existants et le contrôle de la progression des espèces ligneuses exotiques et envahissantes. C'est un parti pris par le Conservatoire du littoral, au regard des données recueillies par les études réalisées sur les savanes réunionnaises.

29 Biotope, Office National des Forêts, Uni Vert Durable, Réalisation d'un plan de gestion de la trame verte et bleue de la commune de Saint-Paul-Phase 1: Diagnostic et définition des enjeux, Commune de Saint-Paul, La Réunion, t. 1, 2014, p. 177. 
Selon ces mêmes études, les savanes ont été façonnées par la présence des troupeaux sur le milieu et par la pratique régulière du feu pastoral par les éleveurs depuis des générations afin de répondre à deux objectifs : redynamiser la ressource fourragère et lutter contre les plantes non consommées par le bétail ${ }^{30}$. Afin de vérifier et de conforter cette idée, le Conservatoire du littoral, avec le concours de l'équipe de recherche, l'APPER, et d'autres acteurs locaux, a mis en place une expérimentation pyro-pastorale sur le site du Cap la Houssaye (Figures 9 et 10), consistant à combiner l'usage du feu et du pâturage, tout en effectuant des suivis scientifiques de leur incidence sur le milieu. Le principal objectif de la mise en place de cette expérimentation pyro-pastorale est de proposer une gestion raisonnée et pérenne de la savane en termes paysagers et de diversité écologique par la mobilisation des deux outils que sont le pâturage et le brûlage dirigé.

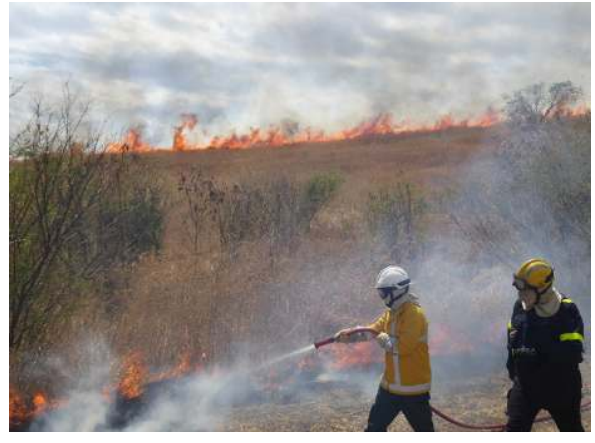

Figure 9 : Opération de brûlage dirigée en août 2018 dans la savane du cap la Houssaye

(C) Quentin Rivière, 2018

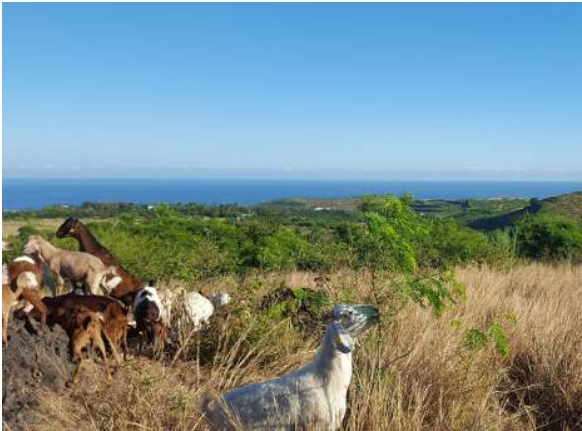

Figure 10 : Troupeau de caprins de l'APPER pâturant dans la savane du cap la Houssaye

(C) Quentin Rivière, 2018

Ainsi, depuis 2016, quatre opérations de brûlage dirigé ont été réalisées. Elles sont conduites en étroite collaboration avec l'État-Major de Zone de Protection Civile de l'Océan-Indien, le Service Départemental d'Incendie et de Secours et l'Office National des Forêts. La mise en place de cette expérimentation a permis de développer des formations de « brûleur » et la création d'une cellule de brûlage dirigé, au sein du SDIS en 2018. Les suivis scientifiques menées sur les dynamiques du feu démontrent une revitalisation des herbacées et la destruction des spécimens ligneux de moins d'un centimètre de diamètre. En revanche le feu semble favoriser la germination des graines des ligneux qui ne sont pas détruits ${ }^{31}$. Ces résultats renforcent l'idée que pour contrôler la prolifération des ligneux il semble nécessaire de coupler l'usage du feu à la pratique du pâturage.

30 Serge Briffaud (éd.) et al., op. cit., p. 191.

31 Pauline Gaud, La végétation de savanes envahies par les ligneux, effets du feu et dynamiques postincendie : le cas des savanes de La Réunion, mémoire de Master 1 Biologie Écologie Évolution, parcours diversité végétale et gestion des écosystème tropicaux, UMR PVBMT-CIRAD UMR Passages, La Réunion, 2017, 52 p. 
S'agissant du pâturage dirigé, l'APPER a initié le projet en 2017, en partenariat avec le Conservatoire du littoral et l'équipe de recherche. L'APPER s'est appuyée sur le troupeau de cabris Péï déjà constitué d'une vingtaine de têtes appartenant à des membres de l'association dans le cadre du projet de sauvegarde de la race Péi. Par la suite, l'objectif a été de créer un troupeau collectif, en mobilisant plusieurs éleveurs adhérents à l'APPER qui ont mis en commun leurs troupeaux respectifs. Dans un premier temps l'association s'est tournée vers un troupeau caprin Péï, en gardant à l'esprit qu’à terme soient mobilisés au sein d'un troupeau mixte des cabris Péï et des bœufs Moka. Au démarrage du projet, quatre éleveurs dont deux éleveurs caprins et deux éleveurs bovinscaprins, contribuaient à la gestion et/ou à l'accompagnement du troupeau au pâturage selon un roulement défini. Depuis 2019, l'APPER a contractualisé avec des bergers pour gérer le troupeau et la conduite au pâturage. Aujourd'hui le troupeau est opérationnel. Il est composé d'une centaine de caprins (boucs, chèvres, chevrettes et petits), qui pâturent 5 jours sur 7 et 4 heures par jour dans la savane. Les autres jours où la conduite au pâturage n'est pas faisable pour des raisons de disponibilités, les animaux sont nourris à l'auge, par les bergers et/ ou les membres de l'association. L'opération de pâturage dirigé fait également l'objet de suivis scientifiques sur le comportement des animaux au pâturage, leurs préférences alimentaires (type, espèce...) et la durée de broutage. Ces informations sont recueillies dans un cahier de pâturage tenu par l'APPER. Ces données permettront d'évaluer le chargement en unité de gros bétail (UGB) caprin/bovin ou mixte envisageable sur la savane en fonction de la saison des pluies et de la saison sèche. Il s'agit également d'évaluer la biomasse (quantité et qualité) disponible dans la savane en fonction des saisons, en réalisant des prélèvements dans la savane et des analyses en laboratoire ${ }^{32}$. Il s'agit également d'étudier l'incidence du broutage sur la végétation herbacée et arbustive de la savane ${ }^{33}$. Enfin, l'objectif est d'évaluer la mise en place de l'opération en fonction du temps, des coûts, des moyens humains (nombres, types, rôles) nécessaires au bon déroulement du projet. Cela a pour finalité de proposer des itinéraires techniques pour une gestion pérenne du site ${ }^{34}$.

La mise en œuvre de ce projet pyro-pastoral répondant à une problématique de gestion paysagère s'inscrit dans un double domaine, reflétant un rapprochement entre le monde agricole et celui de la protection de la nature, en intégrant à la fois des réflexions liées à la diversité agricole et à la diversité naturelle.

32 Suivi piloté par Olivia Fontaine, Zootechnicienne de l'UMR SELMET-Cirad.

33 Suivi piloté par Claudine Ah Peng, Écologue de l'UMR PVBMT, Université de La Réunion et Vincent Boullet, Phytosociologue.

34 Suivi piloté par l'APPER et le CDL. 


\section{De l'individualité à la mise en commun des projets}

L'objectif initial de l'APPER est avant tout la sauvegarde des races animales locales. S'en suit l'intérêt de sauvegarder les pratiques associées à ces races, notamment le pâturage extensif sur les espaces de savane. L'APPER s'est donc investie dans le projet de pâturage dirigé, voyant là une opportunité pour la consolidation d'un troupeau de sauvegarde de cabris Péi et de bœufs Moka, sur leur territoire historique pastoral : la savane.

Quant au Conservatoire du littoral, son objectif initial est la conservation des paysages ouverts de savane. Le pâturage s'avérant être une des modalités de gestion pour le maintien de ce type de paysage d'après les études menées, le CDL s'intéresse donc aux potentiels éleveurs ou groupes d'éleveurs voulant s'installer sur le site du Cap la Houssaye. Cela, sous certaines conditions de pratiques : contrôlées, extensives (parcours), respectueuses de l'environnement et de l'intégrité du site protégé. Au regard des études réalisées sur les savanes, des enquêtes menées auprès des éleveurs des bas de l'Ouest, et des objectifs de l'APPER, l'intérêt du CDL pour le cabri Péí et le bœuf Moka étant des races rustiques et adaptées au milieu, apparait comme pertinent dans le contexte de la savane.

Notons ici que la question de la conservation ou de la mobilisation d'activités agricoles n'est pas nouvelle pour le Conservatoire du littoral. À La Réunion, sur dix-huit sites protégés, cinq sont tout ou partie occupés par des activités agricoles. Elles sont autorisées sur les propriétés du CDL principalement à des fins d'outil de gestion. Sur le site du Cap la Houssaye, le pâturage dirigé est donc davantage perçu par le CDL comme un moyen de gestion du milieu, plutôt qu'un projet agricole. La mobilisation du pâturage n'est pas spécifique au site du Cap la Houssaye. Sur le site de l'étang du Gol protégé depuis 1985 par le CDL, dans le sud-ouest de l'île, certaines zones du site sont entretenues par le pâturage de bovin de race mixte (Moka/autres races métropolitaines). En effet, avant la mise en gestion de ce site, une famille d'éleveur installée sur des parcelles voisines de celles du CDL, faisait pâturer leur troupeau sur le site. Le Conservatoire du littoral, au regard des problématiques d'envahissement sur le site par des espèces exotiques venant freiner le développement des espèces indigènes ou endémiques, a décidé de conserver la pratique de pâturage sur certaines zones pour maintenir une pression sur les espèces exotiques. À la différence de la situation sur le cap la Houssaye, ici, l'éleveur est propriétaire foncier au droit des terrains du CDL, ce qui lui permet d'avoir un système d'exploitation agricole sur son terrain, tout en faisant pâturer ses animaux sur les terrains du CDL. Au Cap la Houssaye, la situation est différente, car le projet a été créé ex-nibilo. Cela a donc nécessité des réflexions et de l'organisation au sujet du transport des animaux, du parc pour le troupeau, de l'accès à l'eau (non existant sur le site), du gardiennage et de la conduite au pâturage. Aujourd'hui le troupeau de l'APPER est accueilli 
(parc, eau) chez un propriétaire privé au droit du site de la savane du cap la Houssaye. Cela engendre des coûts de fonctionnements pour l'association qui, en comparaison de l'éleveur de l'étang du Gol qui est dans une filière de vente d'animaux, n'a pas de rendus financiers liés à l'activité d'élevage. Le troupeau est pour le moment un troupeau de sauvegarde. C'est là-dessus que repose une partie de l'originalité et la complexité de ce projet de pâturage dirigé.

Le projet de pâturage dirigé présenté dans cet article, vient de la rencontre entre deux projets. Celui de la conservation de la savane par une gestion pyropastorale porté par le Conservatoire du littoral et celui de la sauvegarde des races locales, porté par l'APPER. La mise en ouvre de ce projet expérimental repose donc sur deux principales opportunités : pour le CDL, la présence d'un troupeau et pour l'APPER, la présence de surfaces pâturables. Le point principal de convergence des projets est donc le pâturage. Mais chacun des acteurs a initialement des intérêts qui ne sont pas forcément liés au projet de pâturage (Figure 11).

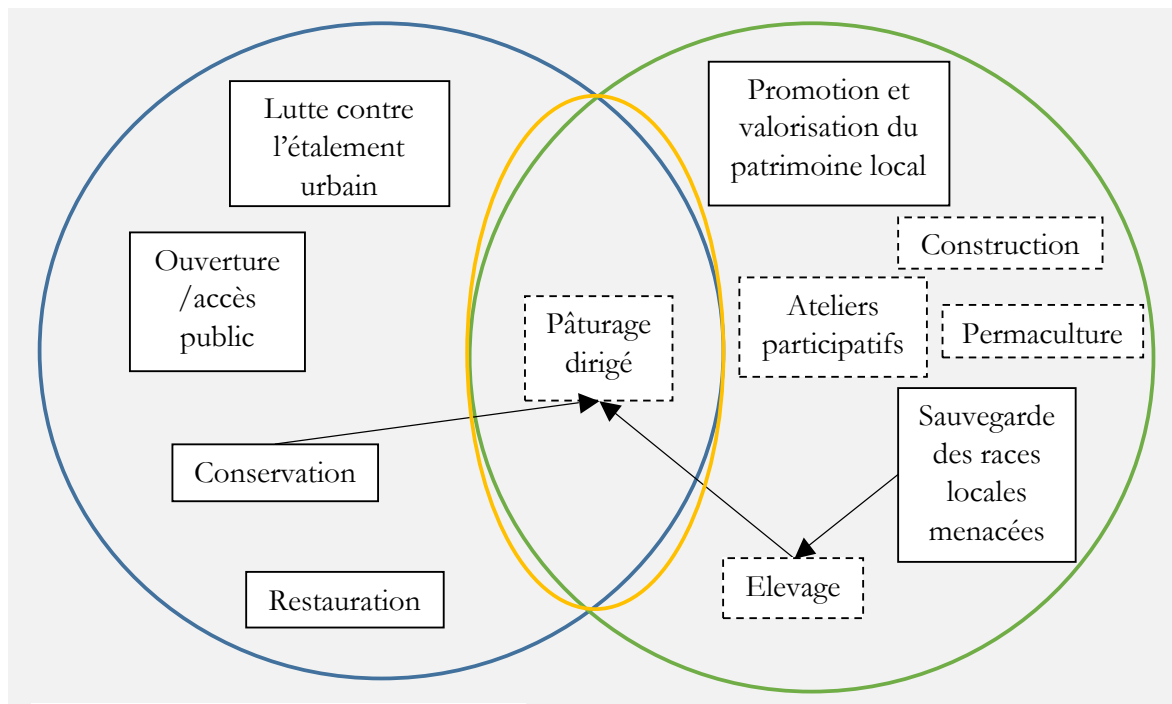

Légende :
- Le Conservatoire du littoral
- L'APPER
- La savane du cap la Houssaye
- Objectifs
-.-.-. Outils

Figure 11 : Des objectifs initiaux différents mais un outil final commun : le pâturage dirigé (C) Quentin Rivière, 2020

Le schéma ci-dessus met en avant la convergence des acteurs vers un outil commun que représente le pâturage dirigé. La mise en place de ce projet a donc permis aux acteurs de se rencontrer et de travailler sur un projet commun tout 
en faisant évoluer les projets respectifs de chacun. D'un côté, le Conservatoire du littoral bénéficie de la présence d'un troupeau sur le site afin de mener les expérimentations de gestion pyro-pastorale. De l'autre, l'APPER bénéficie du soutien du CDL, d'un espace pâturable et avance sur son projet de sauvegarde des races locales en menant des suivis sur le troupeau caprin.

Si l'on croise les objectifs des deux acteurs, nous pouvons voir que, même si les trajectoires sont différentes, nous arrivons à faire converger leurs objectifs (Figure 12).

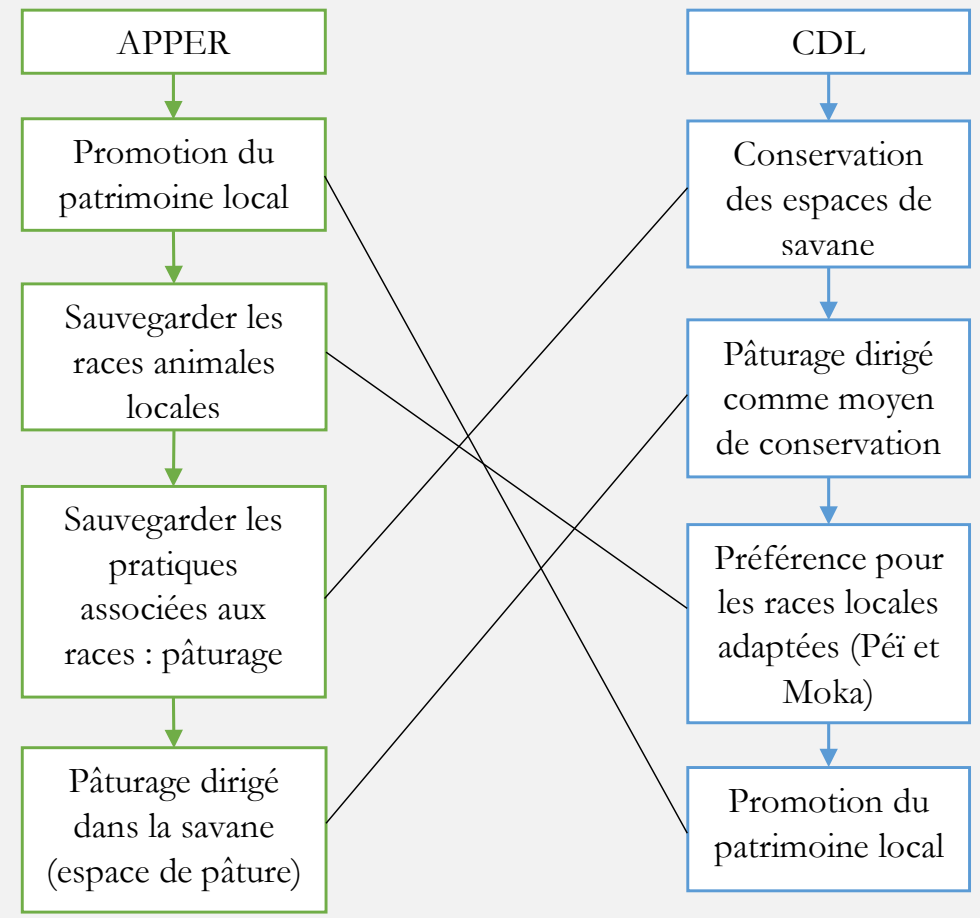

Figure 12 : Les trajectoires d'objectifs croisées entre l'APPER et le CDL

(C) Quentin Rivière, 2020

La convergence de ces projets permet également de mettre en lumière les éleveurs et les bergers de l'île qui pratiquent le pâturage extensif. Car, si l'hypothèse quant à l'utilisation combinée du pâturage et du feu comme outil de conservation efficace des savanes réunionnaises est confirmée, le Conservatoire $\mathrm{du}$ littoral et les gestionnaires d'espace de savane porteront une attention particulière au développement d'activités pastorales sur leurs sites protégés. Ainsi par la mise en ouvre des études et du projet expérimental sur le site du Cap la Houssaye, les éleveurs de bœufs Moka et de cabris Péï, pendant longtemps marginalisés et oubliés, voient leur situation changer. Leurs pratiques 
deviennent des outils de gestion d'intérêt écologique et paysager, et ainsi d'intérêt général et d'utilité publique. Les éleveurs/bergers constituent des acteurs clés dans le cadre de la mise en place d'une gestion pâturée.

Notons cependant que malgré l'étroit lien entre les projets, ils peuvent également évoluer indépendamment l'un de l'autre.

En effet, si nous retournons à l'origine du projet de l'APPER, sur la sauvegarde des races locales, avant même la rencontre avec le Conservatoire du littoral, l'association avait déjà pour ambition de créer un troupeau de sauvegarde de race locale caprine et bovine. Le troupeau caprin était déjà formé d'une vingtaine de tête et était élevé chez un membre de l'association dans les mi-pentes au sud de l'île. Le troupeau bénéficiait d'un abri et d'un parc clôturé où les animaux pouvaient paître à leur guise. Ainsi, si l'on considère la savane comme étant le milieu où ont évolué et où sont habituellement présentes les races locales bovines et caprines réunionnaises, nous pouvons évoquer l'exemple précédent comme modalité de conservation ex-situ, qui consiste à élever des individus d'une espèce hors de leur milieu " naturel ». Dans le cadre strict de la sauvegarde des races animales locales, il semble donc possible de porter le projet hors des espaces de savane. Or aujourd'hui, comme le troupeau conservatoire est élevé en savane, on parlera plutôt d'une conservation in-situ. Cela a pour avantage de pouvoir sauvegarder et conserver les activités pastorales telles que le pâturage extensif dans les savanes, et ainsi la pratique de parcours libres.

Du côté du projet du Conservatoire du littoral quant à l'utilisation du pâturage comme outil de gestion paysagère, il ne dépend pas fondamentalement des races animales locales. En effet, le CDL souhaite maintenir une activité pastorale sur le site de la savane du cap la Houssaye, quelle que soit l'espèce et la race mobilisée. L'objectif est avant tout la gestion paysagère. À titre d'exemple, si l'on reprend le cas du site de l'Étang du Gol, les bovins mobilisés ne sont pas exclusivement de race locale. Le Conservatoire du littoral est ainsi ouvert à toutes propositions d'éleveurs qui souhaiteraient s'installer sur le site du Cap la Houssaye, sous des conditions de période d'essai et de suivis de l'impact du pâturage sur le milieu. L'avantage observé dans le partenariat réalisé avec l'APPER, tient surtout au fait de soutenir une action locale de sauvegarde du patrimoine que représentent les races animales locales. À cela s'y ajoute le caractère rustique de ces races, raison principale de leur mobilisation à ce jour.

Par ces autres trajectoires développées, nous percevons le caractère sensible et fragile de la pérennité du projet commun consistant à la mobilisation d'un troupeau conservatoire de race locale comme outil de gestion des paysages de savanes. Cela ne remet cependant pas en cause la réussite du projet, qui jusqu'aujourd'hui a été dépendante des opportunités qui se sont présentées, de la volonté et de l'implication des différents acteurs concernés. 
Dans cet article nous observons le caractère original de la mise en place d'une gestion des paysages par un troupeau de races animales locales. Le projet de pâturage dirigé présenté dans le présent article se fonde en réalité sur la coexistence et la combinaison de deux projets qui se développent simultanément : le projet de sauvegarde des races animales locales porté par l'APPER, un acteur associatif local, et le projet de gestion du paysage par des pratiques pyro-pastorales, porté par le Conservatoire du littoral, un acteur national avec une assise locale.

Le site protégé de la savane du cap la Houssaye apparait comme un territoire d'expériences et d'apprentissages, tant dans le domaine de la protection et des moyens de conservation de la nature, que dans le domaine agro-environnemental.

La réussite des projets individuels et surtout du projet commun et de sa pérennité tiennent plus d'une volonté des acteurs concernés, que d'un besoin mutuel ou d'une dépendance. On observe donc le passage d'un projet non collectif, à un projet plus collectif, mais plus ambitieux. Cela permet de développer plus d'opportunités (multiplication d'acteurs, financements, valorisation et sensibilisation), mais également d'ajouter des obstacles ou des limites d'actions pour les acteurs (respect des objectifs communs, avancement des projets, réglementations). Il s'agit aujourd'hui d'initier un réseau d'acteurs en vue de développer ces projets.

Les ambitions de ces acteurs sont de créer dans un avenir proche, un conservatoire de races animales locales qui contribueraient à la gestion des espaces naturels protégés à La Réunion. Ce projet est un réel projet de développement local, car il permet et encourage également les éleveurs à s'impliquer dans une démarche collective pour la conservation d'espaces et d'espèces.

\section{Quentin Rivière}

Doctorant Cifre en Sciences Humaines et Sociales, mention Géographie, Université de La Réunion, Océan-Indien : Espaces et Sociétés (OIES) Chargé de projet « Pratiques pastorales », Conservatoire du littoral, Délégation Outre-Mer, Antenne de La Réunion q.riviere@consevatoire-du-littoral.fr

Diplômé d'un master de recherche en Géographie à l'université de La Réunion, je suis actuellement doctorant en sciences humaines et sociales, en contrat CIFRE au Conservatoire du Littoral. Je suis inscrit à l'École Doctorale "Sciences Humaines et Sociales » - ED 541 et rattaché à l'unité de recherche Océan Indien : Espaces et Sociétés (O.I.E.S.) - EA-12. Mon sujet de thèse propose d'étudier et d'interroger les dynamiques territoriales qui résultent de l'utilisation de pratiques pastorales dans la gestion des espaces naturels protégés, avec une application à la savane du Cap La Houssaye, site protégé du Conservatoire du littoral à La Réunion. 


\section{Résumé}

En 2017 une expérimentation de pâturage dirigé a été initiée, avec un troupeau de chèvres Péí, race caprine locale réunionnaise, pour contribuer à la conservation de la savane du cap la Houssaye, protégée par le Conservatoire du littoral sur la commune de Saint-Paul à La Réunion. L'opération est portée par l'Association pour la Promotion du Patrimoine et de l'Écologie à La Réunion (APPER), qui œuvre depuis plusieurs années à la sauvegarde des races animales locales. Cet article fait état de la mise en œuvre de ce projet expérimental. Nous analysons ainsi l'originalité et la singularité de cette démarche à La Réunion et nous interrogeons les conditions de sa réussite et de sa pérennité.

Mots-clés

Sauvegarde, Race locale, Caprin, Pâturage, Savane, espace protégé, projet expérimental.

\section{Abstract}

In 2017, an experimental project of pasture was initiated, with a herd of "Peï" goats, a local goat breed from Reunion Island, to contribute to the conservation of savannah areas, protected by Le Conservatoire du littoral in Saint-Paul municipality in Reunion Island. The operation is carried by the Association for the Promotion of Heritage and Ecology in Reunion Island, which has been working for many years to save the local animal breeds in Reunion Island. This article reports on the implementation of this experimental project. We will analyze the originality and the uniqueness of this project in Reunion Island and we will question the conditions for its success and sustainability.

\section{Keywords}

Perserv, local breed, goat, pasture, savannah, protected space, experimental project. 